\title{
Investigación Clínica Descripción de elementos de radioprotección y dosimetría en Unidades de Cardiología Intervencionista Sudamericanas: Un estudio piloto.
}

Sergio Ramos-Avasola ${ }^{1, a, d,}$ José Uribe ${ }^{2, a}$, Fernando Orsi ${ }^{3, a}$; Tamara Alarcón ${ }^{4, f,}$ José Álvarez ${ }^{4, f}$, Franco de Angelis $4, f$, Jorge Gamarra ${ }^{7, b, e}$, Alex Mora ${ }^{6, a, c}$, Alejandro Prieto ${ }^{5, a}$, Maximiliano Godoy ${ }^{8, a}$, Bernardo León ${ }^{9, a}$, Alicia Tapia ${ }^{10, a}$, Isabel Robles ${ }^{11, a}$, Víctor Arredondo ${ }^{12, a}$, Tomás Aguilar $^{13, a}$, Francisco Vergara ${ }^{14, a}$, Dorothy Sabini ${ }^{115,} g$.

1 Universidad de Viña del Mar, Viña del Mar, Chile, Facultad de Ciencias de La Salud, Escuela de Tecnología Médica, Rodelillo, Viña del Mar. Chile 2 Instituto Cardiovascular de Guadalajara, México.

3 Universidad del Salvador, Argentina

4 Universidad Mayor, Santiago, Chile, Facultad de Medicina, Escuela de Tecnología Médica. 5 Universidad Santo Tomás, Chile, Facultad de Medicina, Escuela de Tecnología Médica. 6 Unidad de Hemodinamia Hospital Dr. Gustavo Fricke, Viña del Mar, Chile. 7 Comisión Chilena de Energía Nuclear, Santiago, Chile.

8 Unidad de Hemodinamia Hospital Carlos Van Burén, Valparaíso, Chile. 9 Unidad de Hemodinamia Hospital de La Serena, Chile. 10 Unidad de Hemodinamia Hospital San Borja, Santiago, Chile 11 Unidad de Hemodinamia Hospital de Concepción, Concepción, Chile. 12 Unidad de Hemodinamia Clínica Tabancura, Santiago, Chile.

13 Unidad de Hemodinamia Hospital de Antofagasta, Antofagasta, Chile. 14 Unidad de Hemodinamia Hospital Clínico de la Universidad Católica, Chile. 15 University of Massachusetts Boston, USA College of Nursing and Health Sciences. a.Tecnólogo Médico, b.Físico, c.Magíster en Estadística, d.Magíster en Epidemiología, e.Magíster en Biofísica Médica, f.Alumnos tesistas, g.Enfermera.

Fuente de financiamiento: Este trabajo no recibió financiamiento de ningún tipo para su realización. Declaración de conflictos de interés: Se declara que, en el presente estudio, no existe conflicto de parte de los autores ya sea personal o institucional. Además, no tiene financiamiento de ninguna empresa de cualquier índole que pudiese alterar la información o datos obtenidos.

Antecedentes: Las unidades de Cardiología intervencional han evidenciado un número creciente de procedimientos, cada vez más variados y complejos, lo cual podría eventualmente generar daños a los profesionales ocupacionalmente expuestos a radiaciones ionizantes (POEs) de no contar con los adecuados elementos de radioprotección y un uso correcto de ellos.

Objetivo: Caracterizar la disponibilidad y utilización de los elementos de radioprotección y dosimetría de unidades de cardiología intervencionista de centros Sudamericanos.

Material y métodos: Se realizó una encuesta autoaplicada a 139 POEs, de ambos sexos de 7 países, a través de una plataforma on-line, se les consultó sobre características demográficas, dosimétricas y de radioprotección.
Resultados: Los elementos de radioprotección más tradicionales; delantales y cuellos plomados se utilizaron un $99,5 \%$ y $98,4 \%$ respectivamente, aquellos elementos más recientes como gafas, gorros y paños plomados solo alcanzaron un $36,8 \%, 6,8 \%$ y $34,2 \%$, de utilización respectivamente, en cuanto a la utilización de los dosímetros, solo un 7,9 \% lo hace apegado a las normas de la Organización Internacional de Energía Atómica (OIEA).

Conclusión: Se constató en la muestra analizada una falta de elementos de radioprotección y un uso inadecuado de ellos, urge realizar intervenciones educativas y técnicas para mejorar estos datos.

Palabras Clave: Radiación, Cardiología intervencional, Equipo de radioprotección, Dosimetría, Profesionales Ocupacionalmente Expuestos.

\section{Correspondencia:}

TM. .Mg. Sergio Ramos Avasola,

sergioramosavasola@gmail.com 


\section{Radioprotection and Dosímetry in Interventional Cardiology Units in South America. A Pilot study}

Background: Due to the increasing number of interventional cardiology procedures currently performed, health professionals (POE) are exposed to ionizing radiation unless adequate protective elements are used.

Aim: to describe the use of radioprotection elements and dosimetry in interventional cardiology laboratories in South America

Methods: A self-administered survey was performed on 139 POE of both sexes and 7 countries using an online platform. Demographic data, dosimetry and characteristics of radioprotection analyzed.

Results: Commonly used radioprotective elements (lead aprons and collars) were used in $99.5 \%$ and 98\% respectively. Recently introduced protection elements like lead goggles, caps and drapery were used in 36.8, 6.8 and 34.2\% , respectively. Dosimetry according to the International Atomic Energy Commission (OIAE) was performed in only $7.9 \%$ of the procedures.

Conclusion: there is a severe lack of adequate radioprotection during interventional cardiology procedures. Urgent measures, including technical implementation and educational interventions are needed to improve radioprotection in interventional cardiology.

Keywords: Radiation, interventional cardiology, radioprotection equipment, professionals occupationally exposed (POE), dosimetry, lead aprons, lead goggles, lead caps, thyroid collar. 


\section{Introducción:}

Los procedimientos en angiografía intervencionista se han vuelto cada vez más frecuentes en la práctica cardiológica y se han incorporado nuevas, diversas y más complejas intervenciones percutáneas, reemplazando los procedimientos quirúrgicos equivalentes, reduciendo así el número de complicaciones y estadías hospitalarias. Sin embargo, esto se ha asociado a una mayor duración de los procedimientos aumentando significativamente la dosis de radiación, tanto para el paciente como también la carga acumulada anual para los profesionales expuestos 1,2 .

Los efectos clínicos producidos por la radiación se clasifican en efectos estocásticos y determinísticos. Los primeros se producen como consecuencia del daño sobre una célula o un pequeño número de células. Ejemplos de efectos estocásticos son los efectos carcinogénicos y los efectos hereditarios de las radiaciones ionizantes. Por su parte, los efectos determinísticos se caracterizan por provocar la muerte de una cantidad de células tal, que no puede ser compensada por la proliferación de células viables. La pérdida resultante de células puede causar deterioros severos de la función de un órgano o tejido, clínicamente detectables. Ejemplos son las cataratas radio-inducidas y las quemaduras por radiación. Los efectos deterministas requieren que se sobrepase un umbral determinado para que se manifiesten, no así en el caso de los efectos estocásticos los cuales ya se podrían manifestar a bajas dosis crónicas de radiación. De hecho, ya hay algunos estudios que describen un exceso de riesgo relativo para muertes por leucemia del orden de 2,96 por cada Gy adicional de exposición ${ }^{3}$ y aterosclerosis de la arteria carótida izquierda ${ }^{4}$. Por lo tanto, deben realizarse esfuerzos para para mantener las dosis más bajas razonablemente posibles (ALARA).

Los materiales que atenúan la radiación, como el plomo, han sido por mucho tiempo utilizados en la confección de elementos de radioprotección (ER) puesto que así se logra reducir la dosis que reciben los profesionales ocupacionalmente expuestos (POE). Estos ER incluyen los tradicionales delantales plomados, que han mostrado ser muy efectivos en bajar la exposición a radiación de los POEs, tanto así que se ha adoptado como una medida estándar en todas las unidades. El inconveniente es su peso puesto que los fabricados con plomo pueden llegar a los 7 kilos $^{5}$, lo que aumentaría el riesgo de problemas a la columna. Los fabricados con otros materiales, como bismuto, solo llegan a 4 kilos manteniendo la misma equivalencia del plomo ${ }^{5}$. Los cuellos tiroideos protegen a la tiroides, un órgano muy radiosensible a efectos carcinogénicos ${ }^{6}$, por lo que siempre deberían usarse. Las protecciones plomadas montadas en el techo, si son correctamente posicionadas pueden bajar las dosis en la cabeza y cuello de los operadores ${ }^{7}$.

Recientemente han aparecido nuevos ER que protegen nuevas zonas a los POEs y pacientes que son atendidos en estas unidades. Entre ellos están las gafas plomadas que están diseñadas para proteger al cristalino, bajando las dosis de radiación hasta en un $98 \%{ }^{8}$. Con ello se evita la formación de cataratas-radioinducidas que se producen habitualmente en las zonas germinativas, específicamente en la superficie anterior donde las células que se dividen forman una fibra-proteica cristalina que migra hacia el polo posterior del cristalino, la región posterior subcapsular. La radiación causa a ese nivel un daño directo y además, mediante mecanismos oxidativos, causa el quiebre de la molécula de ADN, migración de células aberrantes y provoca complejos cambios bioquímicos que dan como resultado proteínas cristalinas aberrantes que provocan finalmente una desregulación de la morfología celular del cristalino ${ }^{9}$. Otro elemento relativamente nuevo son los gorros plomados que se diseñaron para proteger al cerebro, una estructura considerada históricamente de baja radiosensibilidad debido a la protección que le confiere la bóveda craneal. Los estudios que han abordado este tópico son escasos ${ }^{10-12}$. En uno de ellos se describió a médicos intervencionistas que han manifestado lesiones tumorales, preferentemente en el hemisferio cerebral izquierdo ${ }^{10}$. Aun cuando no se demostró que la exposición a radiación ionizante en forma constante sea la causa de esas lesiones, tampoco se descartó dicha posibilidad. En cuanto a la efectividad de los gorros con materiales radio protectores $(\mathrm{Pb} \mathrm{0,5}$; sulfato de bario-óxido de bismuto equivalente a $0,5 \mathrm{~mm}$ de $\mathrm{Pb}$ ), demostraron ser efectivos en reducir la radiación ${ }^{13,14}$. El último ER que ha ingresado al mercado son los denominados paños plomados, que están destinados a disminuir las dosis de radiación secundaria que recibe el POE. Consisten básicamente en un paño estéril fabricado con elementos como bismuto y tungsteno, que una vez colocado sobre el paciente atenúa la radiación secundaria emitida por él en los procedimientos angiográficos. Además, no contiene plomo ni vinilo, pero su densidad y espesor equivalen a $0.25 \mathrm{~mm}$ de plomo, es ligero y según datos del fabricante atenúa alrededor del $95 \%$ en la radiación ionizante secundaria ${ }^{15}$. Sin embargo, en un estudio independiente este dispositivo solo logro reducir en un $23 \%$ la dosis de radiación recibida 
por el POE ${ }^{16}$.

El propósito de este estudio es documentar las condiciones de radio protección y dosimetría en las condiciones reales de trabajo de los POEs que laboran en unidades de cardiología intervencional de una muestra Sudamericana, con el fin de aportar datos concretos para la elaboración de futuras estrategias tendientes a mejorar las actuales condiciones de trabajo en que laboran los POEs.

\section{Materiales y Métodos:}

Esta investigación se adhirió a los principios éticos de la declaración de Helsinki y fue aprobada por la Universidad Mayor. Se realizó una encuesta vía web auto llenada de carácter multicéntrica y multinacional Para iniciarla se debió contar con el apoyo logístico y contactos de dos profesionales Tecnólogos Médicos, quienes ayudaron con la identificación y reclutamiento de los potenciales sujetos de investigación.

Se explicó a los sujetos en detalle la naturaleza de la investigación y se les informó que no se divulgaría información confidencial tanto del personal como de la institución. Una vez aceptadas las condiciones, se les invitó a responder una encuesta online, a través de internet en la plataforma formularios de Google Docs., que plataforma permite realizar encuestas gratuitas online accesibles desde cualquier parte del mundo.

La encuesta estaba formada por 23 preguntas. Se debía consignar información sobre la edad, sexo, años de experiencia en el centro de imagenología, estamento profesional, cantidad promedio de exámenes en que participa el POE, elementos de protección radiológica, tipo y número de dosímetro(s) utilizado(s) junto con la ubicación de estos, Además, indicando la posición de trabajo del POE dentro de la sala de hemodinamia y por último, se preguntó si el centro estaba adscrito a algún sistema de vigilancia epidemiológica de dosis para "Pacientes".

Los datos fueron tabulados inicialmente en una planilla Excel y luego se realizó un análisis estadístico mediante el software SPSS, Chicago, IL; versión 17. En el caso de aquellos centros de hemodinamia en los que se logró evaluar más de un miembro del equipo multidisciplinario que participó en los procedimientos de intervencionismo cardiaco (Médicos, Tecnólogos Médicos, Enfermeras y Técnicos Paramédicos) se promediaron los datos obtenidos. Los valores continuos se expresaron como la media \pm desviación estándar (SD) y los datos categóricos son expresados frecuencia absoluta y $\%$.

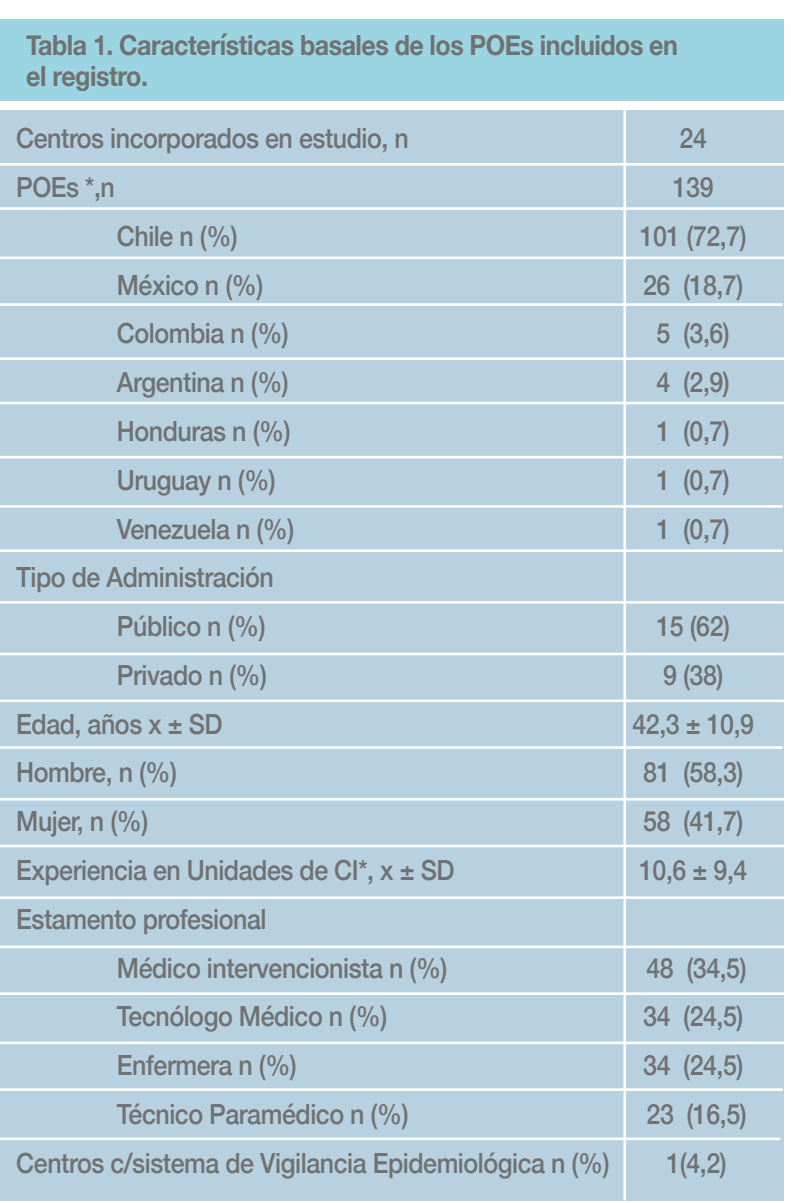

POEs*: Profesionales ocupacionalmente expuestos, $C I^{* *}=$ Cardiología .

\section{Resultados:}

En la Tabla 1 se describen las características de los 139 POEs reclutados en el estudio perteneciente a 24 centros. La mayoría provienen de Chile (9), seguido de México (5). En cuanto a la composición de los equipos de trabajo que laboran en estas unidades, solo México no trabaja con Técnicos Paramédicos, pues en ese país solo se trabaja con Enfermeras, Tecnólogos Médicos y Médicos.

En la Figura 1 se describe el porcentaje de utilización de cada uno de los elementos de radio-protección tradicionales (delantal, cuello tiroideo y gafas plomadas).

En términos globales, en esta muestra, los POEs que trabajan en estas unidades Sudamericanas, utilizan en un $99,5 \%$ los delantales, un 98,4\% los cuellos tiroideos y en un $42,4 \%$ las pantallas acrílicas plomadas.

En la Figura 2, se describe el porcentaje de utilización de cada uno de los elementos de radio protección desarollados y comercializados más recientemente, como: las Gafas plomadas, Gorros y los paños plomados. En términos globales, los POEs que trabajan en estas uni- 


\begin{tabular}{|c|c|c|}
\hline \multirow{2}{*}{\multicolumn{3}{|c|}{$\begin{array}{l}\text { Dosimetría } \\
\text { Tipo }\end{array}$}} \\
\hline & & \\
\hline & Fílmico n (\%) & $21(15,1)$ \\
\hline & OSL n (\%) & $42(30,2)$ \\
\hline & TLD $n(\%)$ & $76(54,7)$ \\
\hline \multicolumn{3}{|l|}{ Cantidad } \\
\hline & $1 \mathrm{n}(\%)$ & $126(90,6)$ \\
\hline & $2 n(\%)$ & $13(9,4)$ \\
\hline \multicolumn{3}{|l|}{ Ubicación } \\
\hline & $\begin{array}{l}\text { a Debajo del delantal plomado a la } \\
\text { altura del pecho } \mathrm{n}(\%)\end{array}$ & $132(95,0)$ \\
\hline & $\begin{array}{l}\text { Sobre el delantal plomado a la } \\
\text { altura del pecho } \mathrm{n}(\%)\end{array}$ & $7(5,0)$ \\
\hline & Debajo protector tiroideo n (\%) & $137(98,2)$ \\
\hline & ${ }^{\mathrm{b}}$ Sobre protector tiroideo $\mathrm{n}(\%)$ & $2(1,8)$ \\
\hline & $a+b n(\%)$ & $11(7,9)$ \\
\hline
\end{tabular}

$a+b$ :Dos dosímetros ubicados uno: debajo del delantal plomado a la altura del pecho y el otro sobre protector tiroideo (Sugerencias de la ICRP y la IAEA).

dades Sudamericanas, utilizan en un 36,8 \% las Gafas plomadas, un 6,8\% los Gorros plomados y un $34,2 \%$ los paños plomados.

Las tecnologías más utilizadas para medir las dosis de radiación son las físicas (Fílmico, TLD y OSL). Las primeras están en franca retirada debido a las múltiples desventajas que presentan comparada con las nuevas tecnologías. Sin embargo, como se puede apreciar en la Tabla 2, estas tecnologías aún están en uso en un 15,1\% de los POEs, en los que predomina el uso de un solo dosímetro. Un 6,8\% aun ubica anatómicamente mal los dosímetros y solo un 7,9\% utiliza en forma correcta los dosímetros en términos de la cantidad, el tipo de tecnología y la ubicación anatómica adecuada.

\section{Discusión:}

Esta investigación es la primera iniciativa conocida a nivel Sudamericano de un estudio multicéntrico que describe como es la realidad de los POEs expuestos a radiación ionizante que laboran en unidades de Cardiología Intervencionista. Su objetivo principal es la eventual realización de intervenciones de Salud Pública destinadas a mejorar las condiciones de radio protección en que laboran dichos profesionales.

Es un hecho que las dosis de radiación en procedimientos intervencionales son las más altas registradas entre el staff médico (Médicos, Tecnólogos Médicos, Enfermeras y Técnicos Paramédicos) que utilizan rayos-X guiados por fluoroscopía 17 . En los Cardiólogos intervencionistas las dosis excederían el límite anual fijado por la ICRP de $20 \mathrm{mSv}$ al año ${ }^{18}$, razón por la cual, estas personas tienen la mayor probabilidad de desarrollar

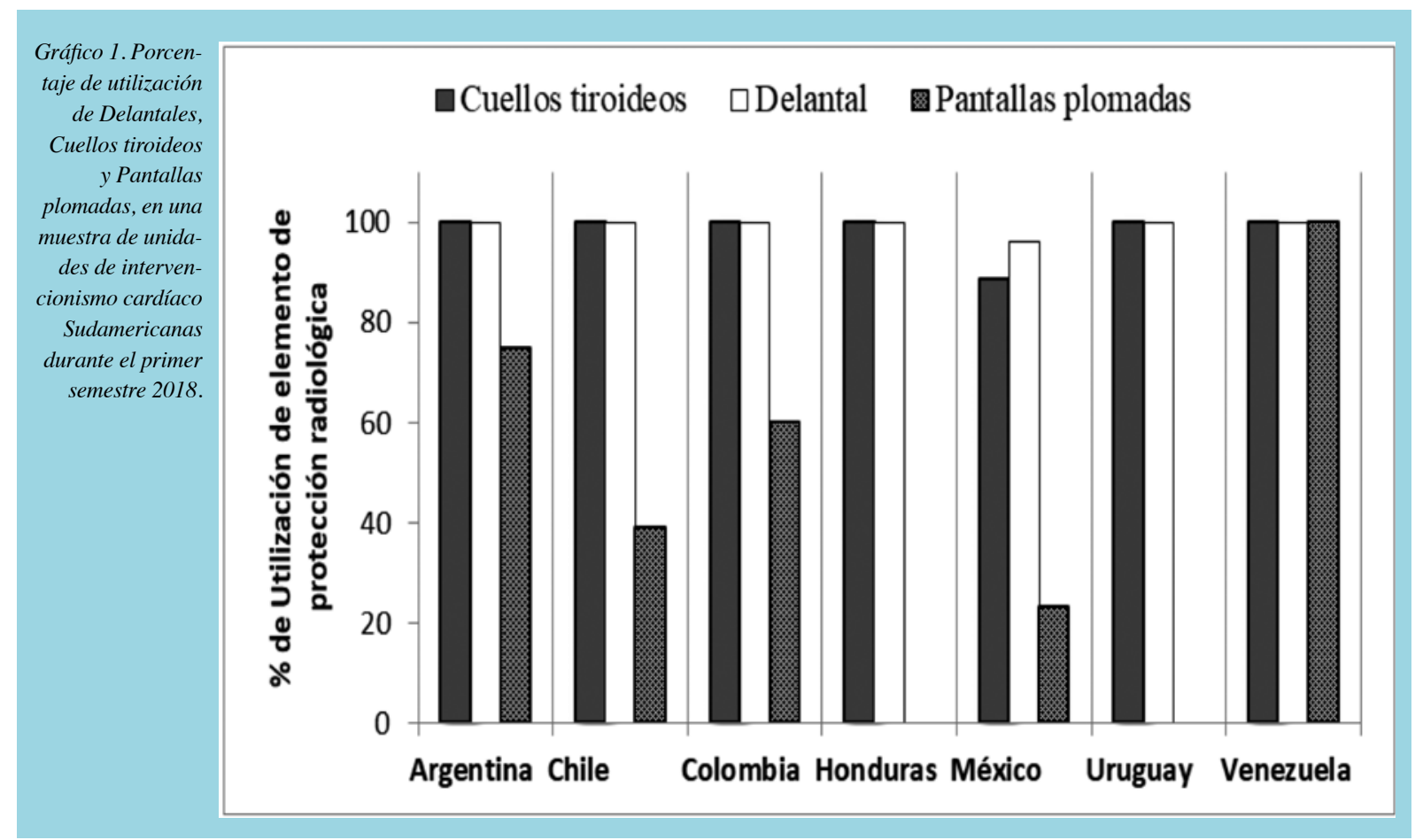




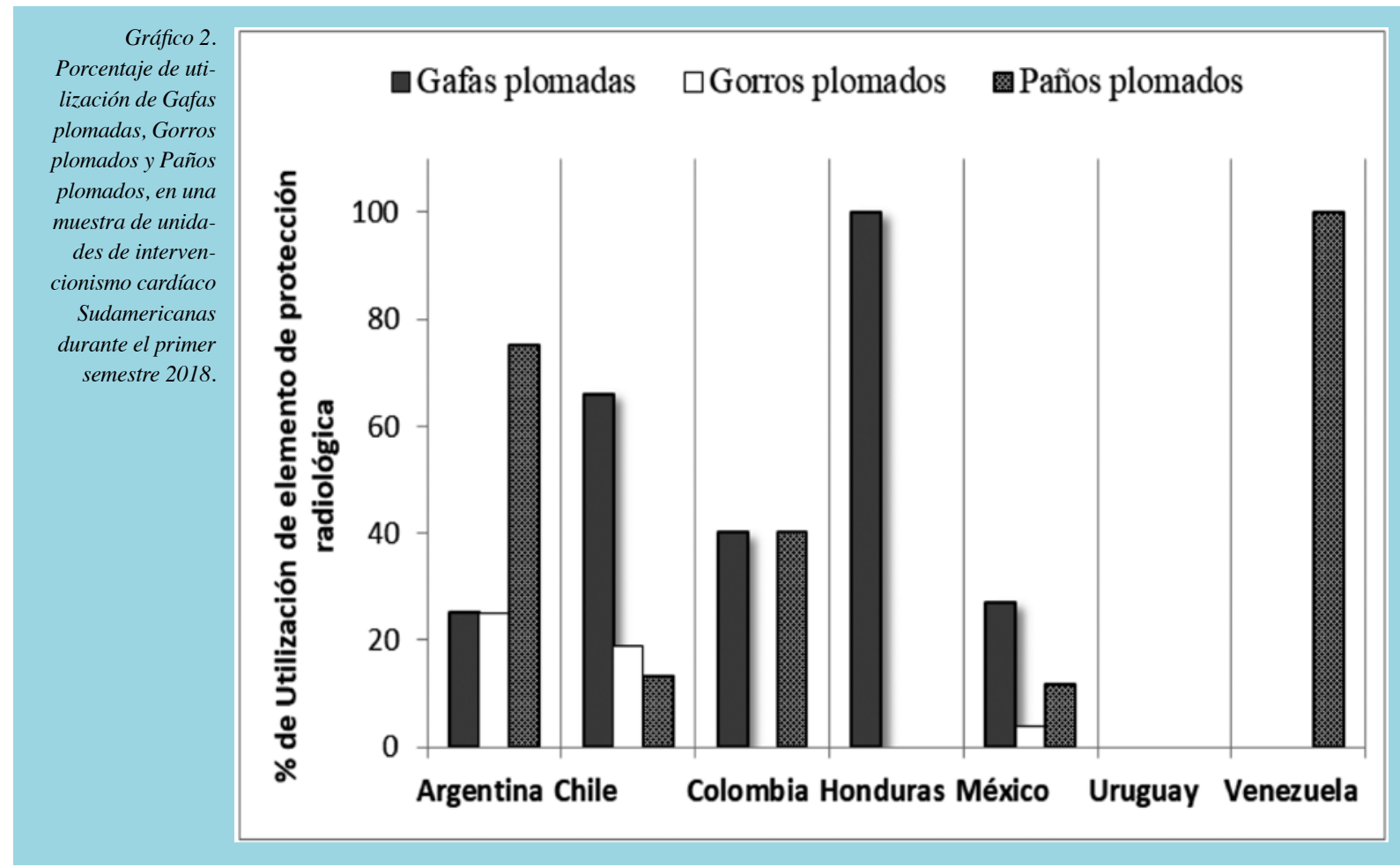

algún evento adverso estocástico o determinístico relacionado con su exposición a los rayos-X. A raíz de lo expuesto, se esperaría que este grupo de profesionales fueran muy rigurosos en el uso de los dispositivos de protección radiológica, pero la práctica clínica dice lo contrario. En esta investigación se constató que, en el mejor de los casos en Chile, sólo se utiliza en promedio el 59,8\% de los ER disponibles; en México la situación es más adversa alcanzando sólo el $40 \%$. Estas cifras no fueron homogéneas En cuanto a delantales y cuellos plomados, ambos son utilizados casi en un $100 \%$ de los POEs encuestados, lo que corrobora que los POEs están conscientes de los beneficios que trae su utilización. En cambio, los ER relativamente nuevos en el mercado (lentes, gorros y paños plomados) se utilizan en baja cantidad. Por ejemplo, los lentes plomados se usaron en promedio, un 36,8\%. En el caso de los gorros plomados un $6,8 \%$ de los POEs lo utilizaron y en el caso de los paños plomados fue un 34,2\%. Ello se debería, probablemente al desconocimiento de los beneficios que traería la utilización de estos nuevos ER. De hecho, hay artículos que han descrito que el uso de lentes plomados disminuyen el efecto de radiación entre 5,7 y 20 veces $^{19-21}$. En Chile, un estudio describió una atenuación de la radiación del orden del $57 \%$ de las dosis recibidas a nivel del cristalino en los POEs de Hemodinamia, producto de la utilización de gafas plomadas con 0,25 mm

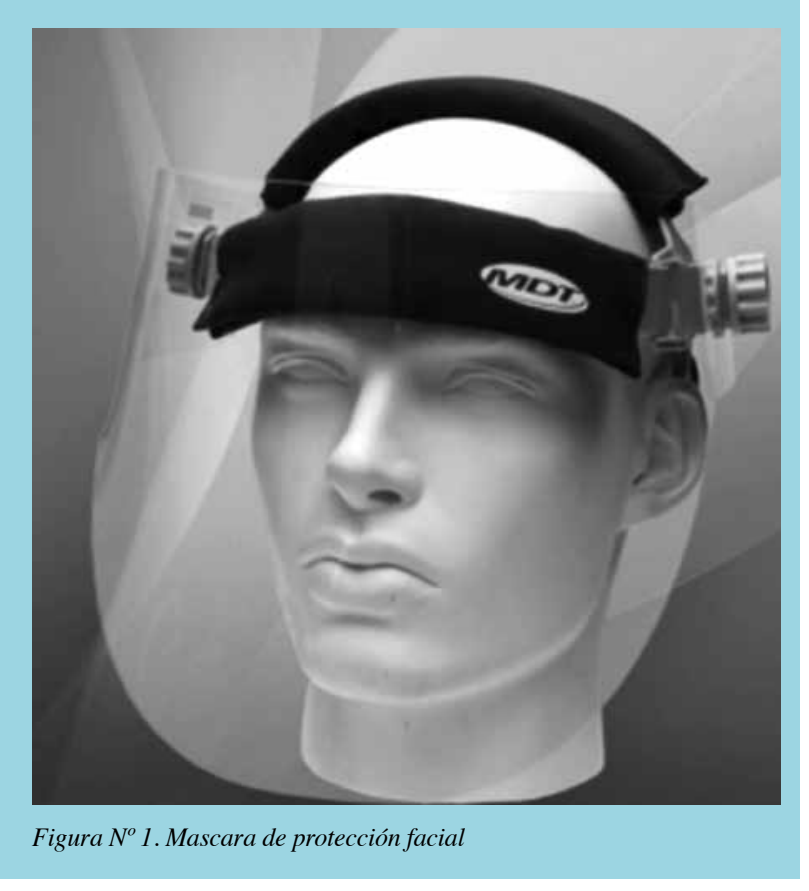

de equivalencia de $\mathrm{Pb}^{9}$. Los gorros plomados reportan una disminución por un factor de $30^{20}$. En Chile 22 se agregó más información indicando que la atenuación observada con gorros plomados de Tungsteno-Bismuto con una equivalencia de $0,125 \mathrm{~mm}$ de $\mathrm{Pb}$, sería de un $40 \%$ en promedio. Sin embargo, esta atenuación sería 
heterogénea según el lóbulo cerebral donde se midió la dosis.

Es importante hacer notar que para la zona ocular y cerebral ha aparecido un nuevo dispositivo denominada mascara plomada de protección facial y ocular (Figura 3). Este dispositivo consiste en una pantalla de acrílico plomada de un peso de 395 gramos Sin embargo en las bases de datos Pubmed, EBSCO, Science direct y Scielo no figuran estudios que den cuenta de su efectividad, por lo que su uso aún no es aconsejable.

En el presente estudio se consultó también sobre la utilización de un nuevo dispositivo de radio protección, destinado a disminuir las dosis de radiación secundaria en el pabellón. Se le conoce como RADPADs y consiste básicamente en un paño estéril fabricado a base de bismuto y tungsteno, que, una vez colocado sobre el paciente, atenúa la radiación secundaria emitida por él en los procedimientos angiográficos. Cabe destacar que no contiene plomo ni vinilo, por lo que es ligero, pero su densidad y espesor equivalen a $0.25 \mathrm{~mm}$ de plomo que, según algunos artículos $18,23-26$, atenuaría en un rango que va de $2 \%$ a $86 \%$. Lamentablemente, a pesar de la evidencia existente a favor de su uso, en la muestra de POEs analizada sólo un 6,8\% lo utiliza, incluso en muchos centros no se utiliza. Sería interesante mediante otro estudio averiguar las razones de fondo que hay detrás de la falta de uso de los nuevos ER que ya cuentan con evidencia sólida sobre su eficacia.

En la práctica clínica, lo habitual es encontrar distintas técnicas dosimétricas, siendo las más frecuentes TLD, OSL y los dosímetros fílmicos. El uso de los dosímetros fílmicos está en descenso porque requieren salas especiales, como cuartos oscuros y salas de revelado. Además, presentan mucha variación entre films y lotes; además, poseen problemas con la dependencia energética $^{27}$. En esta investigación esto quedó evidenciado, pues solo un $21 \%$ sigue utilizando dicha técnica. Es importante considerar, además de la técnica dosimétrica a utilizar, algunos aspectos logísticos que son igual o incluso más relevantes que la técnica en sí; por ejemplo, muchos dosímetros al momento de la lectura están dañados, se extravían, no son devueltos, no son usados, incluso con los resultados que indican cifras "normales" no se podría asegurar que los dosímetros fueron correctamente utilizados. Además del tipo de dosímetro y los aspectos logísticos, se debe considerar la cantidad de dosímetros a utilizar y la ubicación de ellos en el cuerpo de los POEs. En esta investigación solo un $7,9 \%$ de los POEs utiliza los dosímetros siguiendo las directrices emanadas de la Comisión Internacional de Protección Radiológica (ICRP) ${ }^{28}$, institución que es la referente mundial en temas de radio protección. Estas sugerencias también las han tomado en cuenta reconocidas sociedades científicas como la Sociedad Europea de Cardiología Intervencional y la Sociedad de Radiología Intervencional $^{29}$, la Sociedad Japonesa de Cardiología ${ }^{30}$ y la Sociedad Latinoamericana de Cardiología Intervencional ${ }^{31}$. Todas han consensuado en sugerir para las unidades de Cardiología y Radiología Intervencional el uso de dos dosímetros, ubicado uno debajo del delantal plomado a la altura del tórax y el otro en el cuello plomado por fuera de este. En virtud de lo señalado, sería importante considerar la incorporación de la técnica denominada Dosimetría Biológica ${ }^{32}$, como un "complemento" de los programas de vigilancia dosimétrica tradicionales para los POEs. Como ventaja, se puede mencionar que este tipo de dosimetría no es afectada por el uso incorrecto de los dosímetros, y toma en cuenta la radiosensibilidad propia de cada individuo. En la muestra encuestada solo un centro declara contar con un sistema de vigilancia epidemiológica enfocado en el paciente. El centro de enfermedades de los estados Unidos (CDC) define a un sistema de vigilancia epidemiológica consistente en la recolección sistemática, análisis e interpretación como un sistema de datos de salud necesarios para la planificación, implementación y evaluación de políticas de salud pública, combinado con la difusión oportuna de los datos a aquellos que necesitan saber". En la mayoría de los países existen estos sistemas de vigilancia, pero tienen algunas falencias como por ejemplo solo se recolecta la información proveniente de los POEs y no se toma en cuenta las dosis que se reciben. Ya es un hecho conocido que los pacientes también están sujetos a riesgos importantes derivados de su exposición artificial a las radiaciones ionizantes de carácter médico, especialmente en el caso de los procedimientos intervencionales cardiacos como coronariografía., procedimiento diagnóstico equivalente a tomarse 350 radiografías de Tórax. La angioplastia, un procedimiento terapéutico destinado a recanalizar las arterias coronarias, equivale a tomarse 750 radiografías de tórax. Es habitual que el procedimiento terapéutico sea realizado inmediatamente después del diagnóstico o dentro de un periodo relativamente corto. Por ello, en un corto periodo de tiempo estos pacientes acumularán la equivalencia a 1100 radiografías de tórax similar a la radiación que recibiría en forma natural un individuo en 9,2 años de su vida. Incluso, hay procedimientos más extremos en términos de 
los niveles de dosis que alcanzan, como es el caso de las Desoclusiones crónicas de arterias coronarias, donde se puede alcanzar una cifra de 5950 radiografías de tórax lo equivaldría a la dosis natural recibida en 49,5 años 33 . Urge seguir el ejemplo de la OIEA (Organización para la Paz y el Desarrollo de la UN) quienes el año 2001 crearon un plan internacional de acción, destinado a proteger de los efectos de las radiaciones ionizantes a pacientes sometidos a procedimientos radiológicos. Se le conoce como SAFRAD ${ }^{34}$ por sus palabras en inglés (Safety in Radiological Procedures) y básicamente es un sitio web donde se registran en forma voluntaria por los médicos o Tecnólogos médicos los niveles altos o gatillantes de radiaciones ionizantes. Esto alimenta una base de datos internacional que está sirviendo para fines educacionales, de investigación y de seguridad radiológica.

\section{Conclusiones:}

Los ER tradicionales como delantales y cuellos tiroideos se utilizan en forma adecuada por parte de los POEs de las unidades encuestadas. Sin embargo, los ER más nuevos en el mercado se usan en forma insuficiente. Con respecto a la dosimetría, urge realizar intervenciones técnicas y de salud pública para cambiar este escenario.

\section{Referencias}

1. Davies HE, Wathen CG, Gleeson F V. The risks of radiation exposure related to diagnostic imaging and how to minimise them. BMJ. 2011;342.

2. PINAR E, ALBARRÁN A, BAZ JA, MAURI J. Actualización en cardiología intervencionista. Rev Española Cardiol. enero de 2009;62:101-16.

3. LEURAUD K, RICHARDSON B, CARDIS E. Risque de leucémie et de lymphome chez les travailleurs du nucléaire. Environnement, Risques et Sante. Leuraud et al. Open Access article distributed under the terms of CC BY-NC-ND; $2015 ; 14(6): 462-4$.

4. ANDREASSI MG, PICCALUGA E, GARGANI L, SABATINO L, BORGHINI A, FAITA F, et al. Subclinical carotid atherosclerosis and early vascular aging from long-term low-dose ionizing radiation exposure: A genetic, telomere, and vascular ultrasound study in cardiac catheterization laboratory staff. JACC Cardiovasc Interv. 2015;8(4):616-27.

5. PAPADOPOULOS N, PAPAEFSTATHIOU $\mathrm{C}$, KAPLANIS PA, MENIKOU G, KOKONA G, KAOLIS D, et al. Comparison of lead-free and conventional x-ray aprons for diagnostic radiology. IFMBE Proc. 2009;25(3):544-6.

6. RON E, LUBIN JH, SHORE RE, MABUCHI K, MODAN B, POTTERN LM, et al. Thyroid Cancer after Exposure to External Radiation: A Pooled Analysis of Seven Studies. Radiat Res. 1995;141(3):259.
7. DONADILLE L, CARINOU E, BRODECKI M, DOMIENIK J, JANKOWSKI J, KOUKORAVA C, et al. Staff eye lens and extremity exposure in interventional cardiology: Results of the ORAMED project. Radiat Meas. Elsevier Ltd; 2011;46(11):1203-9.

8. THORNTON RH, DAUER LT, ALTAMIRANO JP, ALVARADO KJ, ST. GERMAIN J, SOLOMON SB. Comparing strategies for operator eye protection in the interventional radiology suite. J Vasc Interv Radiol. Elsevier Inc.; 2010;21(11):1703-7.

9. ZETT-LOBOS C, VERA-MUÑOZ F, ARRIOLA-ALVAREZ $\mathrm{K}$, DÍAZ-RAMOS O, GAMARRA J, FERNÁNDEZ-PALOMO C, et al. ¿Es suficiente la protección otorgada por gafas plomadas en cardiología intervencionista? Rev Med Chil. 2013;141:63-9.

10. ROGUIN A, GOLDSTEIN J, BAR O, GOLDSTEIN J A. Brain and neck tumors among physicians performing interventional procedures. Am J Cardiol. Elsevier Inc.; mayo de 2013;111(9):1368-72.

11. ROGUIN A, GOLDSTEIN J, BAR O. Brain tumours among interventional cardiologists: a cause for alarm? Report of four new cases from two cities and a review of the literature. EuroIntervention. enero de 2012;7(9):1-4.

12. KARADAG B, IKITIMUR B, DURMAZ E, AVCI BK, CAKMAK HA, COSANSU K, et al. Effectiveness of a lead cap in radiation protection of the head in the cardiac catheterisation 
laboratory. EuroIntervention. 2013;

13. UTHOFF H, QUESADA R, ROBERTS JS, BAUMANN F, SCHERNTHANER M, ZAREMSKI L, et al. Radioprotective lightweight caps in the interventional cardiology setting: A randomised controlled trial (PROTECT). EuroIntervention. 2015;11(1):53-9.

14. UTHOFF H, PEÑA C, WEST J, CONTRERAS F, BENENATI JF, KATZEN BT. E. AM J ROENTGENOL. 2013;200(4):915-20.

15. WORLDWIDE INNOVATIONS \& TECHNOLOGIES I. Top Xray Radiation Protection Shields \&amp; Caps I Medical Device Manufacturer.

16. POLITI L, BIONDI-ZOCCAI G, NOCETTI L, COSTI T, MONOPOLI D, ROSSI R, et al. Reduction of scatter radiation during transradial percutaneous coronary angiography: A randomized trial using a lead-free radiation shield. Catheter Cardiovasc Interv. 2011;79(1):97-102.

17. SHORROCK D, CHRISTOPOULOS G, WOSIK J, KOTSIA A, RANGAN B, ABDULLAH S, et al. Impact of a Disposable Sterile Radiation Shield on Operator Radiation Exposure During Percutaneous Coronary Intervention of Chronic Total Occlusions. J Am Coll Cardiol. American College of Cardiology Foundation; 2015;65(10):A1779.

18. IQTIDAR AF, JEON C, ROTHMAN R, SNEAD R, PYNE $C T$. Reduction in operator radiation exposure during transradial catheterization and intervention using a simple lead drape. Am Heart J. Mosby, Inc.; 2013;165(3):293-8.

19. KUON E, GÜNTHER M, GEFELLER O, DAHM JB. Standardization of Occupational Dose to Patient DAP Enables Reliable Assessment of Radiation-Protection Devices in Invasive Cardiology. RoFo Fortschritte auf dem Gebiet der Rontgenstrahlen und der Bildgeb Verfahren. 2003;175(11):1545-50.

20. E KUON, J BIRKEL, M SCHMITT JBD. Radiation exposure benefit of a lead cap in invasive cardiology. 2003;1205-10.

21. van Rooijen BD, de Haan MW, Das M, Arnoldussen CWKP, de Graaf R, van Zwam WH, et al. Efficacy of Radiation Safety Glasses in Interventional Radiology. Cardiovasc Intervent Radiol. 2013;37(5).

22. RAMOS-AVASOLA S, DÍAZ N, ROLDÁN R, GAMARRA J, CATALÁN M. ¿Es eficiente la protección anti-radiación otorgada por gorros de pabellón de tungsteno-bismuto en cardiología intervencionista? Rev Med Chile. 2016;144:837-43.

23. MURPHY JC, DARRAGH K, WALSH SJ, HANRATTY CG. EFFICACY OF THE RADPAD Protective Drape During Real World Complex Percutaneous Coronary Intervention Proce- dures. Am J Cardiol. noviembre de 2011;108(10):1408-10.

24. IRANI Z, ALEXANDER B, ZHANG D, LIU B, GHOSHHAJRA B, OKLU R. Novel lead-free drape applied to the X-ray detector protects against scatter radiation in the angiography suite. J Vasc Interv Radiol. Elsevier; 2014;25(8):1200-8.

25. TAMBORINO G, VARGAS CS. Effectiveness of radiation protective drape to reduce exposure in fluoroscopically-guided interventional procedures. 2016;90.

26. SHARMA D, RAMSEWAK A, MANOHARAN G, Spence MS. Efficacy of RADPAD ${ }^{\circledR}$ protection drape in reducing radiation exposure to the primary operator during Transcatheter Aortic Valve Implantation (TAVI). Minerva Cardioangiol. febrero de 2016;64(1):41-6.

27. AGENCY INTERNATIONAL ATOMIC ENERGY. Radiation Dosimeters. En: E.B. Podgorsak, editor. Radiation Oncology Physics Handbook. Viena; 2005. p. 696.

28. VALENTIN J. ABSTRACT: Avoidance of radiation injuries from medical interventional procedures, ICRP Publication 85. Ann ICRP. 16 de junio de 2000;30(2):7-7.

29. MILLER DL, VAÑÓ E, BARTAL G, BALTER S, DIXON R, PADOVANI R, et al. Occupational radiation protection in interventional radiology: A joint guideline of the cardiovascular and interventional radiology society of Europe and the society of interventional radiology. J Vasc Interv Radiol. Elsevier Inc.; 2010;21(5):607-15.

30. JCS Joint Working Group. Guidelines for Radiation Safety in Interventional Cardiology (JCS 2006). Circ J. 2010;74(12):2760-85.

31. DURÁN A, HIAN SK, MILLER DL, LE HERON J, PADOVANI R, Vano E. Recommendations for occupational radiation protection in interventional cardiology. Catheter Cardiovasc Interv. 2013;82(1):29-42.

32. Aires B. Dosimetría citogenética en trabajadores de la salud ocupacionalmente expuestos a radiaciones ionizantes. 2015;

33. BADEL AE, RICO-MESA JS, GAVIRIA MC, ARANGO-ISAZA D, HERNÁNDEZ CHICA CA. Radiación ionizante: revisión de tema y recomendaciones para la práctica. Rev Colomb Cardiol. Sociedad Colombiana de Cardiología y Cirugía Cardiovascular; 2018;(xx).

34. IAEA. Safety in Radiological Procedures (SAFRAD) I IAEA [Internet]. [citado 18 de junio de 2018]. Recuperado a partir de: https://www.iaea.org/resources/rpop/resources/databases-and-learning-systems/safrad. 